\title{
Monotonicity Between Phase Angles and Power Flow and Its Implications for the Uniqueness of Solutions *
}

\author{
SangWoo Park and Richard Y. Zhang \\ and Javad Lavaei \\ University of California, Berkeley \\ $\{$ spark111,ryz,lavaei\}@berkeley.edu
}

\author{
Ross Baldick \\ University of Texas at Austin \\ baldick@ece.utexas.edu
}

\begin{abstract}
This paper establishes sufficient conditions for the uniqueness of power flow solutions in an AC power system via the monotonic relationship between real power flow and the phase angle difference. More specifically, we prove that strict monotonicity holds if the angle difference is bounded by the steady-state stability limit in a power system with a series-parallel topology, or if transmission losses are sufficiently low. In both cases, a vector of voltage phase angles can be uniquely determined (up to an absolute phase shift) given a vector of active power injections within the realizable range. The implication of this result for classical power flow analysis is that, under the conditions specified above, the problem has a unique physically realizable solution if the phasor voltage magnitudes are tightly controlled.
\end{abstract}

\section{Introduction}

Power flow analysis seeks to compute the voltages and flow of power in an AC electric power system, given specific terminal conditions at each system bus. The standard technique is to model the power system as a nonlinear function known as the power flow equations, which maps from a set of $n$ complex voltage phasors to a set of $n$ complex nodal power injections, and to solve for the former given the latter using a variant of Newton's method. Our ability to accurately and reliably perform this computation fundamentally underpins every aspect of power systems: from day-to-day operations in

\footnotetext{
* The authors affiliated with UC Berkeley were in part supported by the ARO grant W911NF-17-1-0555, NSF CAREER Award, and NSF 1807260 . Ross Baldick was in part supported by the University of Texas Faculty Development Program. Due to the space restrictions, the proofs of Theorems 3 and Lemmas 49 have been moved to the technical report [1].
}

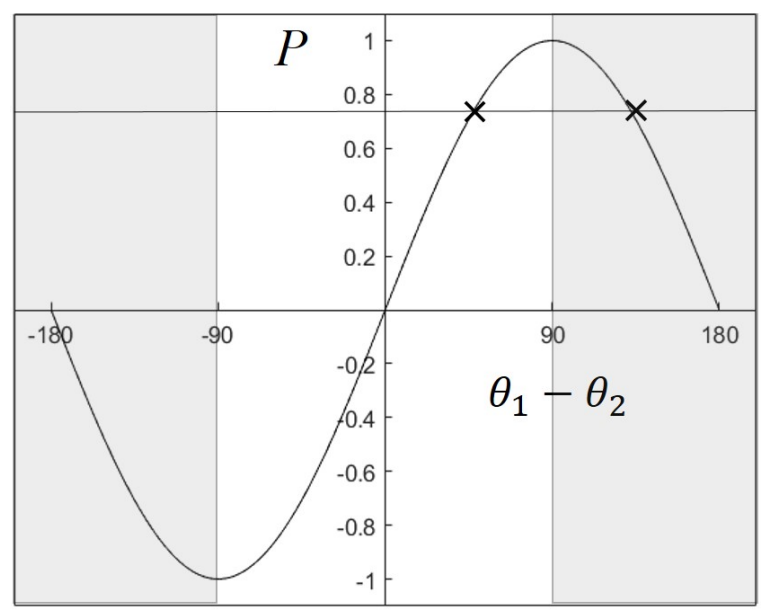

Figure 1: The power flow across a lossless line is a sinusoidal function of the angular difference. This relationship is a strictly increasing function over the angular difference range of $\left|\theta_{1}-\theta_{2}\right|<90$ degrees; a solution restricted to this range is guaranteed to be unique.

contingency analysis and the security-constrained dispatch of electricity markets, to yearly capacity planning for peak load, to decades-long transmission expansion and renewable integration.

The power flow equations are nonlinear, and as such may admit multiple solutions. This poses a significant issue for the system operator, because power flow analysis may converge to a different solution than the one realized by the actual system. There is an extensive body of literature that attempts to understand the nature of multiple solutions, and to eliminate them by restricting the solution to "realistic" values. It has been shown that multiple solutions may persist even after restricting voltage magnitudes to "reasonable values" [2, 3]. In [4, Section IV], the authors provide two solutions to a system with PQ buses, with both solutions having ac- 
ceptable voltage magnitudes within $10 \%$ of the nominal value. However, examples in the literature of spurious solutions tend to violate steady-state stability limits, meaning that they cannot be physically realized.

At the same time, multiple solutions may exist when angular differences are restricted to be less than their steady-state stability limits. Overbye [5] describes a simple two-bus example that admits a high-voltage solution within standard operating limits, and a lowvoltage solution with an unacceptably large phase angle difference that is still below the steady-state limit of 90 degrees. An important point illustrated by Overbye's example is that both power flow solutions are physically realizable, even though only one would be considered acceptable by the system operator. Indeed, the highvoltage solution may give the operator a false sense of security that masks the risk of an impending brown-out due to the low-voltage solution.

\subsection{Monotonicity between phase angles and power flow}

There are reasons to believe that restricting both voltages and angular differences within "realistic" values is enough to force a single, unique solution. Let us illustrate this point on a simple two-bus, one-line lossless system, with line reactance $X$. Here, the transfer of power between the two buses is given with respect to the two voltage magnitudes $V_{1}, V_{2}$ and the angular difference $\theta_{1}-\theta_{2}$ as a sinusoid

$$
P=\frac{V_{1} V_{2}}{X} \sin \left(\theta_{1}-\theta_{2}\right),
$$

illustrated in Figure 1. To simplify the corresponding power flow problem, let us set one bus to be PV while the other as slack. This way, both voltage magnitudes $V_{1}$ and $V_{2}$ are fixed, and the only variable to be solved is the angular difference $\theta_{1}-\theta_{2}$.

Even in this simple toy example, we can see that the power flow solutions are not unique: every value of $P$ can be attained by two different choices of $\theta_{1}-\theta_{2}$. For example, every positive value of $P$ is attained by a choice of $\theta_{1}-\theta_{2}$ below 90 degrees, and another above 90 degrees. Of these two possible solutions, only the one with $\left|\theta_{1}-\theta_{2}\right|<90$ degrees is steady-state stable, and hence physically realizable. The other solution can be viewed as fictitious in the sense that it cannot be sustained without system collapse.

If we restrict $\theta_{1}-\theta_{2}$ to take on physically realizable values within the steady-state stability limit of $\left|\theta_{1}-\theta_{2}\right|<90$ degrees, then the solution becomes unique. Indeed, this follows from the fact that $P$ is strictly increasing with respect to $\theta_{1}-\theta_{2}$ within this range. Formally, if we define $f(x)=\left(V_{1} V_{2} / X\right) \sin x$ as the power flow function and $\Omega=[-\pi / 2,+\pi / 2]$ as the range of acceptible values for $x$, then the strictly increasing property of $f$ guarantees the following inequality

$$
(f(x)-f(y))(x-y)>0 \quad \forall x \neq y, \quad x, y \in \Omega .
$$

The inequality forces the nonlinear equation $f(x)=P$ to have no more than one solution $x \in \Omega$, because a different $y \in \Omega$ satisfying $f(y)=P$ would yield $(f(x)-$ $f(y))(x-y)=0$, thereby contradicting the inequality. Hence, the phase angles $\theta_{1}, \theta_{2}$ can be uniquely determined (up to an absolute phase shift) given a value of $P$ within the realizable range $|P| \leq V_{1} V_{2} / X$.

\subsection{Main results}

Our main contribution in this paper is a generalization of the above approach to the $n$-bus case. The $n$-dimensional analog of a strictly increasing function is a strictly monotone function, which satisfies

$$
(f(x)-f(y))^{T}(x-y)>0 \quad \forall x \neq y, \quad x, y \in \Omega .
$$

If $f$ is strictly monotone over $\Omega$, then the nonlinear equation $f(x)=P$ must have a unique solution $x \in \Omega$ if it does exist.

Analogous to the two-bus case, a set of phase angles are physically realizable for a lossless system if the angular difference across every line $(k, \ell)$ lies within the stability limit of $\left|\theta_{k}-\theta_{\ell}\right|<90$ degrees. This limit will change for the lossy network and is defined for lossy networks in Section 4 . Our two main results in this paper establish the strict monotonicity property between physically realizable phasor angles and the active power bus injections in a lossy power network:

1. When the system topology is a series-parallel graph. Loosely speaking, these are graphs that can be constructed entirely out of series and parallel terminal connections in circuit theory. Any tree graph is a series-parallel graph, as well as any loop. Under the additional condition that the choice of slack bus is to a certain extent flexible, the authors show that strict monotonicity holds for series-parallel graphs. In this case, the monotonicity property arises because the flow of power across series and parallel terminal connections is monotonic with respect to the angular difference across the terminals.

2. When transmission losses are small. In particular, we prove that all lossless transmission networks are monotonic, irrespect of their exact topology. As transmission losses are increased by increasing the resistive component of line admittances, 
the monotonicity property is perturbed until it is no longer guaranteed to hold. The exact amount of losses tolerable is related to the connectivity of the network, the largest line reactance, and the maximum number of branches connected to a single bus (i.e. the maximum degree of the system graph).

In both cases described above, a vector of voltage phase angles can be uniquely determined (up to an absolute phase shift) given a vector of active power injections within the realizable range.

The implication of this result for classical power flow analysis is that, under the conditions specified above, the problem has a unique physically realizable solution if the phasor voltage magnitudes are tightly controlled. This occurs, for example, by imposing PV constraints at all buses, except the slack bus. More generally, we expect power flow analysis with both PQ and PV buses would have a unique solution if tight nominal voltage limits are placed at each bus, say \pm 0.1 per unit. In practice, tightly controlled voltage magnitudes are enforced by operating limits, and are usually achieved through the availability of dispersed and controllable reactive sources. The assumption is implicit in the DC power flow equations.

\subsection{Related work}

Power flow analysis is a classical problem in electric power engineering and there have been many efforts at better understanding the solution set to this problem. In some of the earliest works, researchers have studied various properties of the power flow solutions. Several interesting global and local properties of the stable power flow solutions are presented in [6], limited to lossless transmission networks. Soon after that, Galiana [7] described a set of linear necessary conditions for the solution of the power flow problem, which helped systematically investigate the problem feasibility. Recently, the authors of [8] studied the power flow problem and its relationship to optimization in tree networks by mainly looking at the injection region of the power network.

The following works are dedicated to characterizing conditions under which the power flow solution exists and is uniqe. Under decoupling assumptions, the authors of [9] derive conditions for existence and uniqueness of the reactive power-voltage problem solution. In [10], Ilic extends the previous results by deriving conditions for the real power-phase angle problem (given real power injections, find the voltage angles) and the reactive power-voltage magnitude problem (given reactive power injections, find the voltage magnitudes) under the same decoupling assump- tions. Other related work on uniqueness and existence of power flow solutions includes [11, 12, 13]. In [12] and [13], a fixed point formulation of the power flow problem is used. A domain around a feasible point is specified and sufficient conditions for a unique solution is derived.

As mentioned in Section 1.2, we consider the real power-phase angle problem in this paper. However, unlike in [10], we do not make the decoupling assumptions because it fails to accurately capture the true physics when transmission lines are not purely inductive. Without making decoupling assumptions, [2] investigates the number of power flow solutions in a radial network and show that, for practical system parameters, the solution always exists and is unique. In [14], the results are extended to unbalanced three-phase distribution networks. Meanwhile, the work in [11] establishes topology dependent upper bounds on the number of power flow solutions. Although the results are interesting and valuable, the bounds are calculated for all $Y$ (admittance matrix) and $s$ (complex power injections) and not very useful for our purposes. In this paper, we characterize a group of network topologies under which there exists a unique power flow solution.

Exploiting monotonicity provides one method of proving existence and uniqueness. The aforementioned work [14], shows the monotonic behavior of voltage magnitudes with respect to load changes in radial threephase systems. Another recent work [15] develops a semidefinite programming based procedure to characterize the domain of voltages over which the power flow operator is monotone. In this paper, we also present a condition for monotonicity that depends on the voltages, connectivity of the network, line properties and security angle difference limits.

\section{Definitions and basic assumptions}

We start with some mathematical notations. $\|x\|_{2}$ denotes the $\ell_{2}$-norm of vector $x, X \succeq 0$ means that the matrix $X$ is Hermitian positive semidefinite, and j denotes the imaginary number $\sqrt{-1}$. The symbols $(\cdot)^{T}$ and $(\cdot)^{H}$ denote the transpose and Hermitian transpose of a vector/matrix, respectively. $|\cdot|$ denotes the magnitude of a complex number (e.g. if $x=a+\mathbf{j} b,|x|=\sqrt{a^{2}+b^{2}}$ ) and $\angle$ denotes the angle (i.e. $\left.\angle x=\tan ^{-1}(b / a)\right)$. $\mathfrak{R}(\cdot)$ and $\mathfrak{I}(\cdot)$ denotes the real and imaginary part of a given scalar or matrix, respectively. The operator $\operatorname{diag}(\cdot)$ returns the diagonal of a square matrix.

For graph notations, let $\mathbb{V}=\{1, \ldots, n\}$ be the set of buses in the system and let $\mathbb{E} \subset \mathbb{V} \times \mathbb{V}$ be a set of lines, with the convention that $(\ell, k) \in \mathbb{E}$ implies that $(k, \ell) \in$ 
$\mathbb{E}$. Also, let $\tilde{\mathbb{V}}$ to be the set of all buses except the slack bus. We write $k \sim \ell$ if bus $k$ is connected to bus $\ell$ and $k \nsim \ell$ if they are not connected. Then, $\mathscr{N}(k)$ denotes the set of buses that are connected to bus $k$. Power system topology is specified by the graph $(\mathbb{V}, \mathbb{E})$ and we assume that this graph is connected. Throughout the analyses, the set of buses is fixed and does not change. However, in some cases, we will modify a system by changing the set of lines. For example, we define a system with lines specified by a set $\mathbb{T} \subset \mathbb{E}$ that has tree topology.

Let $v=\left(v_{1}, \ldots, v_{n}\right)^{T} \in \mathbb{C}^{n}$ be the vector of bus voltages and $i=\left(i_{1}, \ldots, i_{n}\right)^{T} \in \mathbb{C}^{n}$ the vector of currents, where $i_{k}$ is the total current flowing out of bus $k$ into the rest of the network. By Ohm's law and Kirchoff's Current Law, the equation $i=Y v$ holds. The complex power injected at bus $k$ is equal to $s_{k}=p_{k}+\mathbf{j} q_{k}=v_{k} i_{k}^{H}$ where $p_{k}$ and $q_{k}$ denote the net real and reactive power injection at bus $k$, respectively. The complex voltage at each bus $k \in \mathbb{V}$ is specified as $v_{k}=V_{k} \angle \theta_{k}=V_{k} \exp \left(\mathbf{j} \theta_{k}\right)$ where $V_{k}$ and $\theta_{k}$ denote the voltage magnitude and phase angle at node $k$, respectively. For convenience, we define $\theta_{k \ell}=\theta_{k}-\theta_{\ell}$ to be the angle difference across the line. Note that, by definition, $\theta_{\ell k}=\theta_{\ell}-\theta_{k}=-\theta_{k \ell}$. Let $p_{k \ell}\left(\theta_{k \ell}\right): \mathbb{R} \rightarrow \mathbb{R}$ be the flow into line $(k, \ell) \in \mathbb{E}$ from bus $k \in \mathbb{V}$ as a function of $\theta_{k \ell}$. Moreover, note that in general $p_{k \ell}\left(\theta_{k \ell}\right) \neq-p_{\ell k}\left(-\theta_{k \ell}\right)$ because of losses in the line. Define $f_{k \ell}$ to be the function relating $p_{k \ell}$ and $p_{\ell k}$. In other words, $f_{k \ell}$ is a function that captures the loss on line $(k, \ell)$ and $p_{\ell k}=f_{k \ell}\left(p_{k \ell}\right)$. Becuase increasing the flow over a line in one direction will decrease the flow in the other direction, it is clear that $f_{k \ell}$ is a strictly decreasing function of $p_{k \ell}$.

Finally, assuming that the shunt elements of the model have zero real part, and assuming that all bus voltages are controlled, we can neglect the admittance of the shunt elements without loss of generality. That is, the reactive power flow into the shunt elements does not affect the uniqueness of the power flow solution.

\section{3. $P-\theta$ problem formulation}

In this section we define our problem formulation and review basic theories behind monotone operators. As mentioned in the introduction, we focus our attention on the relationship between the voltage phasor angles and the real power injections. To this end, we will be considering the mapping from angles to real powers.

Let the bus with index 1 to be the slack bus (also the reference bus) unless defined otherwise. We assume that the voltage magnitudes at all buses, $V=$ $\left(V_{1}, \ldots, V_{n}\right)^{T}$, and $\theta_{1}$ are fixed parameters, and we also assume that the net real power injection is fixed and specified at all buses except the slack bus. We will denote the specified real power injection vector as $\tilde{p}=\left(p_{2}, \ldots, p_{n}\right)^{T}$. The unknown variable is $\tilde{\theta}=$ $\left(\theta_{2}, \ldots, \theta_{n}\right)^{T}$. Although we assume that the voltage magnitude is specified at all buses, we make no assumption about the particular magnitude. For example, the magnitude could be low as in the two bus example described in Section 1 .

In the development below, we will consider a single fixed choice of bus numbers $\mathbb{V}$, but will vary the set of lines $\mathbb{E}$ considered in the system. Consequently, although the specification of the power system depends in general on the choice $(\mathbb{V}, \mathbb{E})$ of both $\mathbb{V}$ and $\mathbb{E}$, we will typically parameterize particular results in terms of $\mathbb{E}$, noting that $\mathbb{V}$ is fixed.

Given a complex bus admittance matrix $Y \in \mathbb{C}^{n \times n}$ and a vector of voltage phasor magnitudes $V \in \mathbb{R}_{+}^{n}$, we can write the equation for the real power injections as:

$$
p_{k}=\Re\left\{s_{k}\right\}=\Re\left\{(Y v)_{k}^{*} v_{k}\right\}
$$

where

$$
v_{k}=V_{k} \exp \left(\mathbf{j} \theta_{k}\right)
$$

Therefore, injection $p$ is only a function of $\theta$ and we can define the following operator.

\subsection{Injection operator}

Definition 1. Let us define $F: \mathbb{R}^{n} \rightarrow \mathbb{R}^{n}$ as the map from the vector of phasor angles to the vector of real power injections:

$$
F_{k}(\theta)=\Re\left\{(Y v)_{k}^{*} v_{k}\right\} .
$$

Also define the truncated injection operator, $\tilde{F}(\tilde{\theta})$ : $\mathbb{R}^{n-1} \rightarrow \mathbb{R}^{n-1}$, as

$$
\tilde{F}(\tilde{\theta})=\left[F_{2}\left(\left[\theta_{1} ; \tilde{\theta}\right]\right), \ldots, F_{n}\left(\left[\theta_{1} ; \tilde{\theta}\right]\right)\right],
$$

where $\theta_{1}$ is a constant (usually assumed zero because bus 1 is the slack/reference bus).

Notice that $\tilde{F}(\tilde{\theta})$ is the operator that describes the $P-\theta$ problem. In the next section, we will see that if an operator $G$ is monotonic over a specified convex domain, then it cannot have multiple solutions.

\subsection{Monotone operators}

Here we will provide a short review on monotone operators. These are all well known results and detailed proofs of the following theorems can be found in [16. [17, 18] and [19, section 2.5.3.] . 
Definition 2. (Monotone operator). A function $G(x)$ : $\mathbb{R}^{n} \rightarrow \mathbb{R}^{n}$ is a monotone operator over a convex domain $\mathscr{D}$ if

$$
\langle G(x)-G(y), x-y\rangle \geq 0 \quad \forall x, y \in \mathscr{D}
$$

and is strongly monotone over $\mathscr{D}$ if

$$
\langle G(x)-G(y), x-y\rangle>0 \quad \forall x, y \in \mathscr{D}, x \neq y
$$

In the case where dimension $n=1$, the above condition for monotonicity is equivalent to the that of a monotonically increasing function: $x \geq y \Longrightarrow G(x) \geq$ $G(y)$. Finally, there is an equivalent characterization of strong monotonicity: A function $G(x)$ is strongly monotone with parameter $m>0$ if

$$
\langle G(x)-G(y), x-y\rangle \geq m\|x-y\|^{2} \quad \forall x, y \in \mathscr{D}
$$

Theorem 1. Suppose that $G$ is strictly monotone over $\mathscr{D}$ and let $c$ be a constant number. Then, the problem of finding $x \in \mathscr{D}$ subject to $G(x)=c$ cannot have multiple solutions.

Theorem 11, stated above without proof, provides the connection between monotonicity and the number of solutions. If we can prove that $\tilde{F}(\tilde{\theta})$ is monotone over a specified convex domain, then we know that the mapping $\tilde{F}: \tilde{\theta} \rightarrow \tilde{p}$ is bijective and therefore the $P-\theta$ problem of finding $\tilde{\theta}$ such that $\tilde{F}(\tilde{\theta})=\tilde{p}$ will have at most one solution.

The following two sections, Section 4 and Section 5 , present the main results of this paper. Section 4 investigates what types of network topologies preserve monotonicity of power flow equations and therefore has a unique solution, if it exists. Section 5 does not explicity consider the network topology. However, by studying the structure of the Jacobian of $F$, we derive conditions under which monotonicity holds.

\section{Monotonicity on networks with series- parallel topologies}

In this section, we ultimately show that under the assumption that voltage angles lie within the steadystate stability limit (which is not very restrictive), monotonicity of the power flow equations holds for all SeriesParallel graphs. As detailed in [20], one of the equivalent definitions of Series-Parallel graphs is as follows:

Definition 3. (series-parallel graphs) A graph is a series-parallel graph if it can be reduced to a single edge graph (graph consisting of two vertices and an edge connecting the two) by a sequence of the following operations:
1. Replacement of a pair of parallel edges with a single edge that connects their common endpoints.

2. Replacement of a pair of edges incident to a vertex of degree 2 with a single edge.

\subsection{Single line properties}

We begin the analysis by looking at a single line. Consider any line $(k, \ell) \in \mathbb{E}$ and its real power flow $p_{k \ell}$ (Figure 2). Elementary calculations show that:

$p_{k \ell}\left(\theta_{k \ell}\right)=G_{k \ell}\left(\left(V_{k}\right)^{2}-V_{k} V_{\ell} \cos \left(\theta_{k \ell}\right)\right)+B_{k \ell} V_{k} V_{\ell} \sin \left(\theta_{k \ell}\right)$

Therefore, given the line properties and the voltage magnitude at both ends, the flow $p_{k \ell}$ depends only on the voltage angle difference $\theta_{k \ell}$. Taking the derivative we get

$$
\left.\frac{\partial p_{k \ell}}{\partial \theta_{k \ell}}\left(\theta_{k \ell}\right)=G_{k \ell} V_{k} V_{\ell} \sin \left(\theta_{k \ell}\right)\right)+B_{k \ell} V_{k} V_{\ell} \cos \left(\theta_{k \ell}\right)
$$

so that $p_{k \ell}$ is strictly monotonically increasing in $\theta_{k \ell}$ if:

$$
\left.G_{k \ell} V_{k} V_{\ell} \sin \left(\theta_{k \ell}\right)\right)+B_{k \ell} V_{k} V_{\ell} \cos \left(\theta_{k \ell}\right)>0 .
$$

Note that $\left.G_{k \ell} V_{k} V_{\ell} \sin \left(\theta_{k \ell}\right)\right)+B_{k \ell} V_{k} V_{\ell} \cos \left(\theta_{k \ell}\right) \geq 0$ if:

$$
-\tan ^{-1}\left(B_{k \ell} / G_{k \ell}\right) \leq \theta_{k \ell} \leq \pi-\tan ^{-1}\left(B_{k \ell} / G_{k \ell}\right),
$$

and that $\left.G_{k \ell} V_{k} V_{\ell} \sin \left(\theta_{k \ell}\right)\right)+B_{k \ell} V_{k} V_{\ell} \cos \left(\theta_{k \ell}\right)=0$ only at the end points of this interval. That is, $p_{k \ell}$ is monotonically increasing in $\theta_{k \ell}$ in this interval. Similarly, $p_{\ell k}$ is monotonically decreasing in $\theta_{k \ell}=-\theta_{\ell k}$ if:

$$
\tan ^{-1}\left(B_{k \ell} / G_{k \ell}\right)-\pi \leq \theta_{k \ell} \leq \tan ^{-1}\left(B_{k \ell} / G_{k \ell}\right) .
$$

Combining these observations, both $p_{k \ell}$ and $p_{\ell k}$ are strictly monotonic functions for:

$$
\left|\theta_{k \ell}\right|<\tan ^{-1}\left(B_{k \ell} / G_{k \ell}\right),
$$

and we will define this region to be the region of steadystate stability of the line $(k, \ell)$ considered individually. That is, if this line were isolated from the system and had generators at each end, then angle differences outside this region will generally result in a destabilizing "swing" in the injection at one or the other or both ends of the line. We will restrict attention to angles that satisfy (6). In what follows, we give the definitions on the steady-state stability limit, allowable angles and set of all possible net injections.

Definition 4. (Allowable sets). Let $\theta \in \mathbb{R}^{n}$ be the vector of angles at all the buses in the system. Also define the steady-state stability limit $\omega_{k \ell}=\tan ^{-1}\left(B_{k \ell} / G_{k \ell}\right)$. Note 


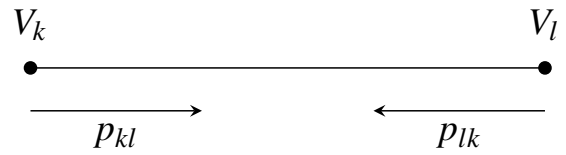

Figure 2: A two-bus network

that $\omega_{k \ell}=\omega_{\ell k}$. For a given power $\operatorname{system}(\mathbb{V}, \mathbb{E})$ we define the set of allowable angles to be:

$$
\Theta(\mathbb{E})=\left\{\theta \in \mathbb{R}^{n}|\forall(k, \ell) \in \mathbb{E},| \theta_{k \ell} \mid<\omega_{k \ell}\right\} .
$$

Furthermore, for a given specification of the lines $\mathbb{E}$ in the system and a given $\theta \in \Theta(\mathbb{E})$, define $\mathscr{P}(\mathbb{E}, \theta) \in$ $\mathbb{R}^{n-1}$ to be the vector of net injections at all the buses except for the slack bus. We define $\mathscr{P}(\mathbb{E}, \Theta(\mathbb{E}))$ to be the set of all possible net injections for allowable angles.

We acknowledge that there is not a one-to-one correspondence between the notion of stability of a line considered individually in isolation and the steady-state and transient stability of an actual power system, particularly where there are additional control feedback loops such as "power system stabilizers." However, limiting angles to satisfy (6) results in some convenient properties of power flow solutions. For example, we state the following elementary result without proof:

Lemma 2. Define $\underline{p}_{k \ell}=p_{k \ell}\left(-\omega_{k \ell}\right)$ and $\bar{p}_{k \ell}=p_{k \ell}\left(\omega_{k \ell}\right)$. Then for each $p_{k \ell}^{\star} \in\left(p_{k \ell}, \bar{p}_{k \ell}\right)$ there exists a unique $\theta_{k \ell}^{\star}$ with $\left|\theta_{k \ell}^{\star}\right|<\omega_{k \ell}$ such that $p_{k \ell}^{\star}=p_{k \ell}\left(\theta_{k \ell}^{\star}\right)$. Moreover, this means that for given $p_{k \ell}^{\star} \in\left(\underline{p}_{k \ell}, \bar{p}_{k \ell}\right)$ there is a uniquely determined corresponding value $p_{\ell k}^{\star}$ of injection at the other end of the line.

\subsection{Tree properties}

In this subsection, we build on the results for a single line to prove uniqueness of the $P-\theta$ power flow problem for the special case where the topology of the network is a tree. We also show that the mapping from the real power injections to voltage angles is monotonic and that the set of all possible net injections for allowable angles (see Definition 4) is a convex set. Although a tree network is not realistic for transmission systems, this will provide important results that will be used for the general case of a mesh. Some of the results that we mention here are already well known in the existing literature. However, we organize the proof of this existing result around the monotonicity property, with the goal of generalizing the same arguments to mesh networks.

We will write $\mathbb{T} \subset \mathbb{V} \times \mathbb{V}$ for a collection of lines that form a tree and consider power systems with graphs $(\mathbb{V}, \mathbb{T})$ that have tree topology. Recall that the reference/slack bus is bus 1. A key observation about tree topology is that for any bus $k \in \mathbb{V}$ there is a unique path $E_{k}=\left\{\left(k, k^{\prime}\right),\left(k^{\prime}, k^{\prime \prime}, \ldots,\left(k^{\prime \cdots \prime}, 1\right)\right\} \subset \mathbb{T}\right.$ of successive lines between bus $k$ and bus 1 , which we consider to be the root of the tree. Define the "distance" $d(k)$ of bus $k$ from bus 1 to be the number of lines in the unique path $E_{k}$ between bus $k$ and bus 1 in the tree $\mathbb{T}$. We define $E_{1}=\emptyset$ and $d(1)=0$. Generically, results in such networks are proved by beginning with leaves and proceeding towards bus 1 using induction on decreasing distance to bus 1 . We have the following theorem:

Theorem 3. Suppose that the power system has graph $(\mathbb{V}, \mathbb{T})$ with tree topology and is connected. Then:

1. for each $\tilde{p} \in \mathscr{P}(\mathbb{T}, \Theta(\mathbb{T}))$ there is a unique $\theta \in$ $\Theta(\mathbb{T})$ such that $\tilde{p}=\mathscr{P}(\mathbb{T}, \theta)$ and $\theta_{1}=0$.

2. $\mathscr{P}(\mathbb{T}, \Theta(\mathbb{T}))$ is a convex set.

Proof. The proof is provided in [1].

Note that by Part 1 of Theorem 3 , for a given power system $(\mathbb{V}, \mathbb{T})$ with tree topology, there is a well-defined function $\vartheta$ such that for each $\tilde{p} \in \mathscr{P}(\mathbb{T}, \Theta(\mathbb{T}))$, the unique value $\tilde{\theta}$ with $\theta=[0 ; \tilde{\theta}] \in \Theta(\mathbb{T})$ and $\tilde{p}=\mathscr{P}(\mathbb{T}, \theta)$ satisfies $\tilde{\theta}=\vartheta(\mathbb{T}, \tilde{p})$. That is, $\vartheta(\mathbb{T}, \bullet)$ is the inverse of $\mathscr{P}(\mathbb{T}, \bullet)$. Moreover $\vartheta(\mathbb{T}, \bullet)$ is differentiable. For each $k, \ell \in \mathbb{V}$, we define $\vartheta_{k \ell}=\vartheta_{k}-\vartheta_{\ell}$. Note that we only require $k, \ell \in \mathbb{V}$ and do not require $(k, \ell) \in \mathbb{T}$ in the definition of $\vartheta_{k \ell}$ and in the following:

Lemma 4. Suppose that the power system $\mathbb{G}=(\mathbb{V}, \mathbb{T})$ has tree topology and is connected. Then, for each $k, \ell \in \mathbb{V}$ and for each $\tilde{p} \in \mathscr{P}(\mathbb{T}, \Theta(\mathbb{T}))$, we have that $\frac{\partial \vartheta_{k \ell}}{\partial p_{k}}(\mathbb{T}, \tilde{p}) \geq 0$.

Proof. The proof is provided in $[1]$.

\subsection{Extension to series-parallel graphs}

By building on the results of the prevoius subsections, we characterize the group of meshed networks for which we can obtain a uniqueness result similar to that of Theorem 3 . We first present an examplary demonstration of our method by proving the uniqueness result for a single cycle. Then we show that outer-cycles do not interfere with the uniqueness and therefore can be "reduced." We conclude that all graphs that are reducible to a tree has a unique powerflow solution. These graphs turn out to be equivalent to a group of graphs called Series-Parallel. However, we do not have a convexity result analogous to Theorem 3 for the mesh case and, indeed, the set of allowable injections for a meshed network may be non-convex.

Example 1. Let $\tilde{p} \in \mathscr{P}(\mathbb{G}, \Theta(\mathbb{G}))$ where $\mathbb{G}$ is a singlecycle network. Then, given the assumptions made in 


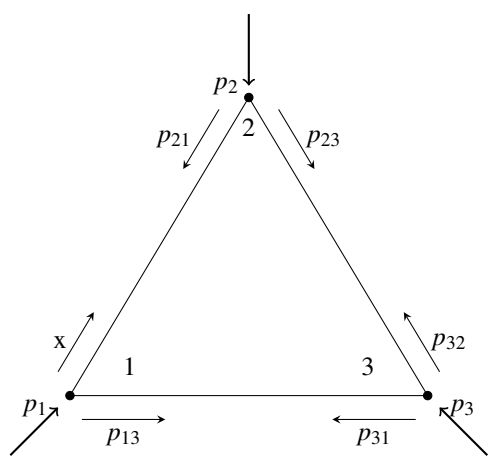

Figure 3: A single-cycle network

section 2, there is a unique solution $\theta \in \Theta(\mathbb{G})$ to the $P-\theta$ problem such that $\tilde{p}=\mathscr{P}(\mathbb{G}, \theta)$ and $\theta_{1}=0$.

Proof. See Figure 3 Without loss of generality, we set node 3 to be the slack bus and denote $p_{12}$ as $x$. This means Then, the following equations hold:

$$
\begin{aligned}
& p_{23}=p_{2}-p_{21}=p_{2}-f_{12}\left(p_{12}\right)=p_{2}-f_{12}(x) \\
& p_{31}=f_{13}\left(p_{13}\right)=f_{13}\left(p_{1}-p_{12}\right)=f_{13}\left(p_{1}-x\right) \\
& \theta_{12}+\theta_{23}+\theta_{31}=0
\end{aligned}
$$

The first two equations are from energy balance and the third equation comes from the fact that the sum of voltage angle difference around a cycle is zero. Since $f_{12}$ and $f_{13}$ are decreasing functions, we can see that $p_{23}$ and $p_{31}$ are monotonically increasing with respect to $x$. Furthermore due to Lemma 2, we know that $\theta_{k \ell}$ is monotonically increasing with respect to $p_{k \ell}$. As a result, $\theta_{12}, \theta_{23}$ and $\theta_{31}$ are all monotonically increasing w.r.t $x$ and therefore equation 9 emits a unique solution.

Moving on, now we will show that the problem of determining the uniqueness of powerflow solution for a meshed network can be reduced to the problem of uniquenss on a smaller subgraph tha excludes the original graph's "outer-cycle," defined below. The final result says that any graph that can be reduced into a tree has unique powerflow solutions. We immediately realise that Example 1 is a special case because a single cycle can be reduced into a single edge, which is a tree. Below is how we define an "outer-cycle."

Definition 5. Let $\mathbb{G}$ be a general meshed network that contains at least one cycle. A cycle $\mathbb{L} \subset \mathbb{G}$ is called an outer-cycle if it shares at most one edge with the rest of the network.

Lemma 5. Let $\tilde{p} \in \mathscr{P}(\mathbb{G}, \Theta(\mathbb{G}))$ where $\mathbb{G}$ is a general meshed network that contains an outer-cycle $\mathbb{L}$. Suppose line $(k, \ell)$ is an edge that is part of the cycle $\mathbb{L}$.

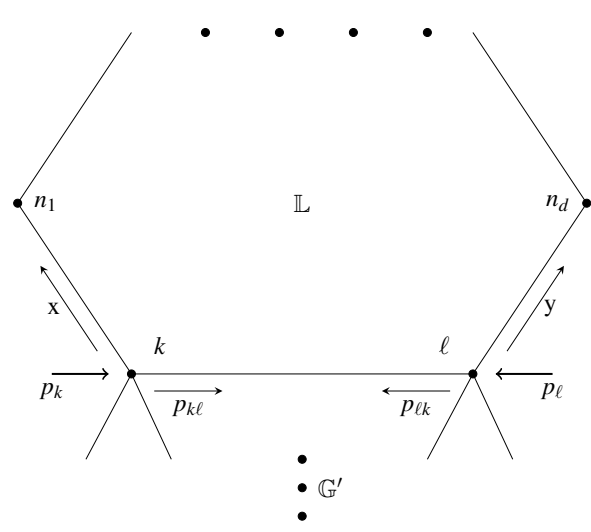

Figure 4: A meshed network with outercycle

Let $\mathbb{G}^{\prime}$ be a subgraph of $\mathbb{G}$ such that $\mathbb{G}^{\prime} \cup \mathbb{L}=\mathbb{G}$ and $\mathbb{G}^{\prime} \cap \mathbb{L}=\ell$. Then,

1. The net power injection from $\mathbb{G}^{\prime}$ into $\mathbb{L}$ is monotonic with respect to the angle difference across $\mathbb{L}$

2. The problem of determining the uniqueness of powerflow solution for $\mathbb{G}$ is reduced to the same problem but for $\mathbb{G}^{\prime}$

Proof. We will prove this by showing that the power flow coming out of the line that is shared by the outercycle and the rest of the network is an increasing function of the power flow going into that line. Without loss of generality, consider Figure 4. Let the outer-cycle $\mathbb{L}$ consist of vertices $k, n_{1}, \ldots, n_{d}, \ell$. The edge connecting nodes $k$ and $\ell$ is the line that is shared between $\mathbb{L}$ and the rest of the graph. The power flow in and out of this line, denoted by $p_{k}^{\prime}$ and $p_{\ell}^{\prime}$, can be described as:

$$
\begin{aligned}
& p_{k}^{\prime}=p_{k}+\text { all other flows from } \mathbb{G}^{\prime} \text { into node } k \\
& p_{\ell}^{\prime}=p_{\ell}+\text { all other flows from } \mathbb{G}^{\prime} \text { into node } \ell
\end{aligned}
$$

Also, as before by energy balance and the cycle rule,

$$
\begin{aligned}
p_{k}^{\prime} & =x+p_{k \ell} \\
p_{\ell}^{\prime} & =y+p_{\ell k} \\
\theta_{k \ell} & =\theta_{k n_{1}}+\theta_{n_{1} n_{2}}+\cdots+\theta_{n_{d-1} n_{d}}++\theta_{n_{d} \ell}
\end{aligned}
$$

Consider $x$ to be the injection into the path $\left(k, n_{1}, \ldots, n_{d}, \ell\right)$ and apply Lemma 4 Then, we see that Equation 12 is monotonically increasing in $x$, which means that $x$ is increasing in $\theta_{k \ell}$. By symmetry we can also show that $y$ is decreasing in $\theta_{k \ell}$. Finally, using equation 10 and 11 , we can see that both $p_{k}^{\prime}$ (the flow into the shared line) and $-p_{\ell}^{\prime}$ (the flow out of the shared line) are increasing in $\theta_{k \ell}$. Therefore, $-p_{\ell}^{\prime}$ is increasing in $p_{k}^{\prime}$. This proves the first part of the lemma. The second part of the lemma follows naturally because once $p_{k}^{\prime}$ is specified uniquely, then via Equation $10, x$ and $p_{k \ell}$ will also be determined uniquely. 
Lemma 5 is useful because it can be used to characterize the group of graphs on which the powerflow solution is unique. In fact, Lemma 5 implies that deleting the graph's outer-cycle does not influence the uniqueness of the power flow solution on that graph. It turns out that the procedure of deleting an outer-cycle is equivalent to the two operations allowed for SeriesParallel graphs as defined in Definition 3 . To show this, suppose there is an outer cycle as laid out in Figure 4. We can make the path $\left(k, n_{1}, \ldots, n_{d}, \ell\right)$ into another direct edge between $k$ and $\ell$ by subsequently applying operation 2 . Then, we apply operation 1 which deletes the outer cycle. Repeating this for every outer-cycle will reduce the original graph to a tree graph. Let's denote this tree graph, reduced by Series-Parallel (SP) operations from the original graph $\mathbb{G}$, as $\mathbb{T}_{S P}(\mathbb{G})$. Finally, since we showed the uniqueness property on tree networks in Section 4.2, and because a tree can be further reduced to a single edge by continuously applying operation 2 , any graph that can be turned into a tree by subsequently deleting outer-cycles, can also be turned into a single edge and in addition will have a unique power flow solution. The only caveat is that we have to assume that the slack bus is selected to be in the tree that was reduced from the original graph. The final result is given in the below theorem.

Theorem 6. Suppose that $\mathbb{G}$ is a Series-Parallel graph. Also, assume that the slack bus is part of the SP-reduced tree graph of $\mathbb{G}$, denoted by $\mathbb{T}_{S P}(\mathbb{G})$. Then, the $P-\theta$ power flow problem cannot have multiple solutions.

\section{Monotonicity on low-loss networks of ar- bitrary topology}

The result in Section 4 applied to lines with arbitrary values of resistance and losses, but only applied to specific topologies. Here, the result is very flexible with topology, but inflexible with losses. In the lossless case, the solution is unique (a folklore result), if it exists. Our result extends and generalizes this to the low-loss case. We will give conditions on $Y$ and $V$ to guarantee that $\tilde{F}$ is strongly monotone up to absolute phase. Under these conditions, $\tilde{F}$ is a bijective map over $\Theta$; the nonlinear system of equations $\tilde{F}(\tilde{\theta})=\tilde{p}$ has a unique solution in $\Theta$. Moreover, it also offers a convergence guarantee for Newton's method with an appropriately chosen step-size rule. We begin with a key lemma.

We present the following main theorem. The theorem derives a lower bound on the strong monotonicity constant $m$. If $m$ is positive, then the (real power) injection operator is strictly monotone and uniqueness is guaranteed.
Theorem 7. Let $Y=Y^{T}$ be complex symmetric. Then, the following holds

$$
\left\langle F(\theta)-F\left(\theta^{\prime}\right), \theta-\theta^{\prime}\right\rangle \geq m\left\|\theta-\theta^{\prime}\right\|^{2} \quad \forall \theta, \theta^{\prime} \in \Theta
$$

over the set of angles

$$
\Theta=\left\{\theta \in \mathbb{R}^{n}: \theta_{1}=\gamma, \quad\left|\theta_{k}-\theta_{\ell}\right| \leq \omega_{k \ell} \quad \forall k \sim \ell\right\},
$$

with strong monotonicity constant

$m=\frac{\alpha}{n} \cdot V_{\min }^{2} \cdot B_{\min } \cdot \cos \delta_{\max }-\operatorname{deg} \cdot V_{\max }^{2} \cdot G_{\max } \cdot \sin \delta_{\max }$

where

- $\gamma$ is a constant;

- $\omega_{k \ell}$ was defined in Definition 4

- deg is the maximum degree of the system graph;

- $\alpha$ is the algebraic connectivity of the system graph (the second-smallest eigenvalue of its unweighted graph Laplacian);

- $B_{\min }$ is the smallest branch susceptance, as in $+\mathfrak{I}\left\{Y_{k, \ell}\right\} \geq B_{\min }$ for all $k \sim \ell$;

- $G_{\max }$ is the largest branch conductance, as in $-\Re\left\{Y_{k, \ell}\right\} \leq G_{\max }$ for all $k \sim \ell$; and

- $V_{\max }, V_{\min }$ are bounds for the voltage magnitudes, as in $V_{\min } \leq V_{k} \leq V_{\max }$ for all $k \in\{0, \ldots, n\}$.

Proof. The proof is provided in the Appendix.

Corollary 8. Let $Y=Y^{T}$ be complex symmetric. Then, the following holds

$$
\left\langle\tilde{F}(\tilde{\boldsymbol{\theta}})-\tilde{F}\left(\tilde{\theta}^{\prime}\right), \tilde{\theta}-\tilde{\theta}^{\prime}\right\rangle \geq m\left\|\tilde{\boldsymbol{\theta}}-\tilde{\theta}^{\prime}\right\|^{2} \quad \forall \boldsymbol{\theta}, \boldsymbol{\theta}^{\prime} \in \Theta
$$

where $\tilde{\theta}$ is defined in Section $3 \tilde{F}(\tilde{\theta})$ is defined in Definition 1 and $m$ is defined in Theorem 7

Proof. Because $\theta_{1}=\gamma,\left\langle F(\theta)-F\left(\theta^{\prime}\right), \theta-\theta^{\prime}\right\rangle=$ $\left\langle\tilde{F}(\tilde{\theta})-\tilde{F}\left(\tilde{\theta}^{\prime}\right), \tilde{\theta}-\tilde{\theta}^{\prime}\right\rangle$ and $\left\|\theta-\theta^{\prime}\right\|^{2}=\left\|\tilde{\theta}-\tilde{\theta}^{\prime}\right\|^{2}$. Plug these equalities into the result of Theorem 7 and the proof is complete.

We see that $\tilde{F}$ is guaranteed to be strongly monotone if the system is lossless. For another approach to proving strong monotonicity of $\tilde{F}$ in the lossless case, see [19, exercise 6.5].

If the system is lossy, then we need $X / R$ ratios to be large, voltages ranges to be small, angular differences to be small, and for the system topology to be wellconnected. The monotonicity of $\tilde{F}$ immediately establishes the goal of this paper; under the assumptions of Theorem 77, if $m>0$, the $P-\theta$ problem cannot have multiple solutions. 


\section{Conclusion}

This paper is concerned with understanding what additional conditions can rule out the possibility of multiple solution for power flow equations. In general, power flow equations may have multiple solutions because of their nonlinearity. To this end, we have established conditions for the uniqueness of power flow solutions (if it exists) in an AC power system via the monotonic relationship between real power flow and the phase angle difference. More specifically, we prove that strict monotonicity holds if the angle difference is bounded by the steady-state stability limit and if either the power system has series-parallel topology, or when there is no restriction on the network topology, if the $X / R$ ratios are sufficiently large, voltage magnitude ranges are sufficiently small, angular differences are sufficiently small, and the network has sufficiently low degree. These conditions guarantee the uniqueness of power flow solution, if it exists. In both cases, a vector of voltage phase angles can be uniquely determined (up to an absolute phase shift) given a vector of active power injections within the realizable range. The implication of this result for classical power flow analysis is that, under the conditions specified above, the problem has at most one physically realizable solution if the phasor voltage magnitudes are tightly controlled.

\section{References}

[1] S. Park, R. Y. Zhang, R. Baldick, and J. Lavaei, "Monotonicity between phase angles and power flow and its implications for the uniqueness of solutions," Technical report archived and available at http://lavaei.ieor. berkeley.edu/HICSS_2019.pdf 2018.

[2] H.-D. Chiang and M. E. Baran, "On the existence and uniqueness of load flow solution for radial distribution power networks," IEEE Transactions on Circuits and Systems, vol. CAS-37, no. 3, pp. 410-416, March 1990.

[3] I. A. Hiskens and R. J. Davy, "Exploring the power flow solution space boundary," IEEE Transactions on Power Systems, vol. 16, no. 3, pp. 389-395, August 2001.

[4] H. D. Nguyen and K. S. Turitsyn, "Appearance of multiple stable load flow solutions under power flow reversal conditions," in Proceedings of the IEEE PES General Meeting, National Harbor, MD, July 2014, available from: https://arxiv.org/pdf/1404.6591.pdf.

[5] T. Overbye, "Lecture Notes: ECEN 460 power systems operation and control," 2017, available from: https://overbye.engr.tamu.edu/course-2/ecen460fa2017/ lecture-notes/

[6] A. Araposthatis, S. Sastry, and P. Varaiya, "Analysis of power-flow equation," Int. Journal of Electrical and Power Energy Syst., vol. 3, pp. 115-126, July 1981.

[7] F. Galiana, "Analytical investigation of the power flow equations," 1983, pp. 411-415.

[8] J. Lavaei, D. Tse, and B. Zhang, "Geometry of power flows in tree networks," IEEE Trans. on Power Syst., vol. 31, pp. 163-172, January 2016.

[9] J. Thorp, D. Schulz, and M. Ilic-Spong, "Reactive power-voltage problem: conditions for the existence of solution and localized disturbance propagation," Int. Journal of Electrical and Power Energy Syst., vol. 9, January 1986.

[10] M. Ilic, "Network theoretic conditions for existence and uniqueness of steady state solutions to electric power circuits," in Proceedings of the IEEE International Symposium on Circuits and Systems, 1992.

[11] T. Chen and D. Mehta, "On the network topology dependent solution count of the algebraic load flow equations," IEEE Transactions on Power Systems, vol. 33, no. 2, pp. 1451-1460, March 2018, available from https: //arxiv.org/pdf/1512.04987.pdf

[12] C. Wang, A. Bernstein, J.-Y. Le Boudec, and M. Paolone, "Explicit conditions on existence and uniqueness of load-flow solutions in distribution networks," IEEE Transactions on Smart Grid, vol. 9, no. 2, pp. 953-962, March 2018.

[13] S. Z. S. Bolognani, "On the existence and linear approximation of the power flow solution in power distribution networks," IEEE Trans. on Power Syst., vol. 31, pp. 163172, January 2016.

[14] K. Miu and H.-D. Chiang, "Existence, uniqueness, and monotonic properties of the feasible power flow solution for radial three-phase distribution networks," IEEE Trans. on Circuits and Syst., vol. 47, pp. 1502-1514, October 2000.

[15] K. Dvijotham, S. Low, and M. Chertkov, "Solving the power flow equations: a monotone operator approach," 2015.

[16] R. Phelps, Convex Functions, Monotone Operators and Differentiability, 2nd ed.

[17] H. Bauschke and P. Combettes, Convex analysis and monotone operator theory in hilbert spaces, 2nd ed.

[18] E. Ryu and S. Boyd, "A primer on monotone operator methods," Appl. Comput. Math, vol. 15, no. 1, pp. 3-43, 2016.

[19] R. Baldick, Applied Optimization: Formulation and Algorithms for Engineering Systems. Cambridge: Cambridge University Press, 2006.

[20] R. Duffin, "Topology of series-parallel networks," Journal of Math. Analysis and Appl., vol. 10, pp. 303-318, 1965.

\section{Appendix}

In the appendix we state Lemma 9, which is the first step to proving Theorem 7 Following the lemma, we provide the proof of Theorem 7

Lemma 9. The Jacobian $\nabla F$ of $F$ satisfies the following

$$
\frac{1}{2}\left(\nabla F(\theta)+\nabla F(\theta)^{T}\right)=\operatorname{diag}(d)+Q^{T} \operatorname{diag}(w) Q
$$


where for each $i$-th edge $(k, \ell)$ with $k>\ell$ we define

$$
\begin{aligned}
w_{i} & =\frac{1}{2} V_{k} \cdot V_{\ell} \cdot\left[\left|Y_{\ell, k}\right| \cdot \sin \left(\theta_{k}-\theta_{\ell}+\angle Y_{\ell, k}\right)\right. \\
& \left.-\left|Y_{k, \ell}\right| \cdot \sin \left(\theta_{k}-\theta_{\ell}-\angle Y_{k, \ell}\right)\right]
\end{aligned}
$$

and for each $k$-th vertex we define

$$
\begin{aligned}
d_{k} & =-\frac{1}{2} \sum_{k \sim \ell} V_{k} \cdot V_{\ell} \cdot\left[\left|Y_{k, \ell}\right| \cdot \sin \left(\theta_{k}-\theta_{\ell}-\angle Y_{k, \ell}\right)\right. \\
& \left.+\left|Y_{\ell, k}\right| \cdot \sin \left(\theta_{k}-\theta_{\ell}+\angle Y_{\ell, k}\right)\right]
\end{aligned}
$$

and $Q$ is the following directed incidence for the graph,

$$
Q_{i, i}= \begin{cases}+1 & \text { edge } i \text { is }(k, \ell) \text { and } k>\ell \\ -1 & \text { edge } i \text { is }(k, \ell) \text { and } k<\ell \\ 0 & \text { otherwise. }\end{cases}
$$

proof. The proof is provided in [1].

\section{Proof of Theorem 7}

Proof. We wish to prove strong monoticity of the injection operator $F$. Let us begin with a key lemma. In the special case that $Y_{k, \ell}=Y_{\ell, k}$ for all $k, \ell \in \mathbb{V}$, the expressions for $w_{i}$ and $d_{k}$ simplify to the following:

$$
\begin{array}{r}
w_{i}=+V_{k} V_{\ell} \mathfrak{I}\left\{Y_{k, \ell}\right\} \cos \left(\theta_{k}-\theta_{\ell}\right) \\
d_{k}=-\sum_{k \sim \ell} V_{k} V_{\ell} \Re\left\{Y_{k, \ell}\right\} \sin \left(\theta_{k}-\theta_{\ell}\right)
\end{array}
$$

This is via the elementary trigonometric identity $\sin (\alpha+\beta)-\sin (\alpha-\beta)=2 \cos \alpha \sin \beta$. By using the simplified expression above, we can derive lower bounds for $w_{i}$ and $d_{k}$, which follow directly from the definitions of deg, $V_{\min }, V_{\max }, B_{\min }, G_{\max }$ and $\delta_{\max }$

$$
\begin{aligned}
& w_{i} \geq V_{\min }^{2} \cdot B_{\min } \cdot \cos \delta_{\max } \\
& d_{k} \geq-\operatorname{deg} \cdot V_{\max }^{2} \cdot G_{\max } \cdot \sin \delta_{\max }
\end{aligned}
$$

Now define $\mu$ as the following:

$$
\begin{aligned}
\mu=\min _{u \in \mathbb{R}^{n}} & u^{T} \nabla F(\theta) u \\
\text { s.t. } & \|u\|_{2}=1 \\
& u_{1}=0
\end{aligned}
$$

We can show that

$$
\begin{aligned}
u^{T} \nabla F(\theta) u & =u^{T}\left[\frac{1}{2}\left(\nabla F(\theta)+\nabla F(\theta)^{T}\right)\right] u \\
& =u^{T}\left[Q^{T} W Q+D\right] u \\
& \geq \min (w) \cdot u^{T} Q^{T} Q u+\min (d)
\end{aligned}
$$

where the second equality follows from Lemma 9 . Note that $Q^{T} \operatorname{diag}(w) Q$ is a graph Laplacian. Its smallest eigenvalue is zero with eigenvector $\mathbf{1}$, and its second smallest eigenvalue is related to its algebraic connectivity $\alpha$ via

$$
Q^{T} Q \succeq \alpha \cdot\left(I-\frac{1}{n} \mathbf{1 1}^{T}\right) .
$$

The lower-bound to Equation 19 can be found by considering the following optimization problem:

$$
\begin{aligned}
& \min _{u \in \mathbb{R}^{n}} u^{T} Q^{T} Q u \quad \text { s.t. }\|u\|_{2}=1 \text { and } u_{1}=0 \\
\geq & \min _{u \in \mathbb{R}^{n}} u^{T} \alpha \cdot\left(I-\frac{\mathbf{1 1}^{T}}{n}\right) u \quad \text { s.t. }\|u\|_{2}=1 \text { and } u_{1}=0 \\
= & \min _{u \in \mathbb{R}^{n}} \alpha \cdot\left[u^{T} u-\frac{\left(\mathbf{1}^{T} u\right)^{2}}{n}\right] \quad \text { s.t. }\|u\|_{2}=1 \text { and } u_{1}=0 \\
= & \alpha \cdot\left[1-\frac{n-1}{n}\right]=\frac{\alpha}{n}
\end{aligned}
$$

Equation 21 comes directly from Equation 20, 22 is due to a simple algebraic manipulation and 23 is because the maximum value of $\left(\mathbf{1}^{T} u\right)^{2}$ subject to $\|u\|_{2}=1$ and $u_{1}=$ 0 is equal to $n-1$. Therefore, when restricted to $\|u\|_{2}=$ 1 and $u_{1}=0$, Equation 19 becomes

$$
u^{T} \nabla F(\theta) u \geq \min (w) \cdot \frac{\alpha}{n}+\min (d)
$$

By taking the infimum on both sides of the inequality, we also get

$$
\mu \geq \min (w) \cdot \frac{\alpha}{n}+\min (d)
$$

Finally, the goal is to show that $F(\theta)$ is strongly monotone by using Definition 2 Taking the results that we have so far, and applying the fundamental theorem of calculus yields

$$
\begin{aligned}
& \left\langle F(\theta)-F\left(\theta^{\prime}\right), \theta-\theta^{\prime}\right\rangle \\
& =\int_{0}^{1}\left(\theta-\theta^{\prime}\right)^{T} \nabla F\left(\theta^{\prime}+t\left(\theta-\theta^{\prime}\right)\right)\left(\theta-\theta^{\prime}\right) d t \\
& \geq \mu \int_{0}^{1}\left\|\theta-\theta^{\prime}\right\|^{2} d t=\mu\left\|\theta-\theta^{\prime}\right\|^{2} \\
& \geq\left[\min (w) \cdot \frac{\alpha}{n}+\min (d)\right]\left\|\theta-\theta^{\prime}\right\|^{2}
\end{aligned}
$$

for all satisfying $e_{1}^{T}\left(\theta-\theta^{\prime}\right)=0$. Plugging in the lower bounds for $w$ and $d$ given in Equation 17 and 18 completes our proof. 